\title{
Physiology of Basketball - Field Tests. Review Article
}

\author{
by \\ Roni Gottlieb', Asaf Shalom², Julio Calleja-Gonzalez ${ }^{3}$
}

\begin{abstract}
The game of basketball is characterized by short and intense bouts of activity at medium to high frequency. Basketball entails specific types of movements, physiological requirements and energy sources. The duration of physiological responses involving ATP, CP and glycolysis responses to this type of activity is 5-6 seconds for a single sprint, and a contribution of the aerobic system is of less than $10 \%$. Recovery periods in basketball, as a rule, are not long enough to fill the gap for such high intensity activities. It is hard to achieve the same level of performance consistently over time in repeated sprints. This means that basketball players need great athletic ability in order to demonstrate speed, strength and power required to produce a successful performance most proficiently. Therefore, tests are needed to help coaches to monitor their players and ensure that they have the physiological capacity required for the game. The aim of fitness tests is to assess the condition of athletes in terms of each fitness component, in order to determine what needs to be improved through the training program and to conduct retests at set times to assess whether their condition has changed. The literature offers a number of widely used tests to measure aerobic and anaerobic fitness. This article reviews the physiological demands of basketball and analyzes the field tests commonly used at present. The article emphasizes the need for a specific test that will serve coaches and physical fitness trainers in monitoring their players.
\end{abstract}

Key words: aerobic, anaerobic, explosive.

\section{Introduction}

The game of basketball is characterized by short and intense bouts of activity at medium to high frequency (Meckel and Gottlieb, 2009; Meckel et al., 2009). Such activity requires aerobic and anaerobic capabilities, both of which impact anaerobic performance (Gottlieb et al., 2014). The ability to continuously perform intermittent highintensity actions throughout the game is crucial for basketball players (Ben Abdelkrim et al., 2007). Thus, higher aerobic capacity has been found to be essential for basketball players' performance in games and in practice (Castagna et al., 2008), in order to recover faster.

Basketball includes high-intensity movements lasting less than $6 \mathrm{~s}$ and moderateintensity exercise of up to $60 \mathrm{~s}$ (Stolen et al., 2005). The duration of physiological responses involving
ATP, CP and glycolysis responses to this type of activity is 5-6 s for a single sprint, and a contribution of the aerobic system is of less than $10 \%$. During recovery from intense activity, when $\mathrm{CP}$ must be replenished, blood lactate concentration is used as a source of energy and phosphates accumulated in the cells are removed (Wragg et al., 2000). For example, in basketball short recovery periods do not last long enough to fill the gap for such high-intensity activities. The ability of basketball players to continue to play well over time depends on rebuilding CP storage and removing waste products - both of which are functions of the aerobic system (Glaister, 2005). Basketball is one of the fastest team sports, and is characterized by exceptional movements such as sprints, changes of direction, dunks, rebounds and blocked shots (Gottlieb et al., 2014). This means

1 - The Academic College at Wingate, Wingate Institute, Netanya, Israel.

2 - The Matte Asher Basketball Club, Matte Asher, Israel.

3 - Physical Education and Sport Department, Faculty of Education and Sport University of the Basque Country, UPVIEHU,

Vitoria-Gasteiz, Spain.

Authors submitted their contribution to the article to the editorial board.

Accepted for printing in the Journal of Human Kinetics vol. 77/2021 in January 2021. 
that basketball players need great athletic ability in order to most proficiently demonstrate speed, strength and power required to produce a successful basketball performance (Delextrat and Cohen, 2008).

The game of basketball has undergone radical changes in the past decade. Coaches believe that the rule changes in May 2000 (Meckel and Gottlieb, 2009; Meckel et al., 2009) that shortened offensive attack time from 30 to $24 \mathrm{~s}$ and the time allowed to cross the median line from 10 to $8 \mathrm{~s}$, as well as subdividing play time into four 10 -min quarters instead of two 20-min halves, modified the tactical and physical demands of the game. Basketball players have been found to cover about 4500-5000 $\mathrm{m}$ during a 48-min game (Crisafulli et al., 2002), and spend only $34.1 \%$ of the time playing, $56.8 \%$ walking, and $9.0 \%$ standing (Narazaki et al., 2009). Thus, identifying the physiological requirements of modern basketball is essential in order to develop and prescribe an appropriate physical training program (Abdelkrim et al., 2007).

Many of the key actions performed by basketball players in a game are based on horizontal movements (sprints and changes of direction), vertical movements (jump shots and rebounds) and combinations of movements within both of these planes, mainly when penetrating to the basket and blocking a shot (Meckel and Gottlieb, 2009; Meckel et al., 2009). These highintensity movements are usually performed intermittently throughout the game (Gottlieb et al., 2014).

The fitness component and energy system in basketball

Many coaches and players equate athleticism with physical fitness in this type of sport. Being physically fit is essential from a health standpoint, but the following fitness components are equally important for elite basketball players (Abdelkrim et al., 2007; Gottlieb et al., 2014; Shaher, 2011): cardiorespiratory fitness, muscular strength, muscular endurance, flexibility and body composition.

The first component, cardiorespiratory fitness, refers to the effective delivery of blood, oxygen and nutrients to the active body by the heart and lungs during physical work. Aerobic exercise improves cardiorespiratory function (Meckel et al., 2009) and also strengthens the heart muscle. Aerobic training can be done through any activity requiring continuous low-intensity effort for 20-60 min (Meckel and Gottlieb, 2009). In this sense basketball requires short and intense periods of activity, during which players expend a great deal of energy at a rapid rate. Anaerobic pathways are another aspect of cardiorespiratory fitness, and provide energy for high-intensity activities. Thus the anaerobic energy systems must also be well developed (Abdelkrim et al., 2007; Gottlieb et al., 2014; McInnes et al., 2008).

The physiology underlying the aerobic and anaerobic energy systems is complex, and especially so in basketball (Gottlieb et al., 2014; Meckel and Gottlieb, 2009). On the one hand, the aerobic system, which supplies long-term energy, depends on the presence of oxygen for the production of ATP. This is the preferred energy source for exercise lasting more than $3 \mathrm{~min}$ (Castagna et al., 2005; Meckel and Gottlieb, 2009; Meckel et al., 2009). When basketball players begin exercising, both the aerobic and anaerobic energy systems are involved. However, the relative contribution of each energy source varies according to the demands of the exercise, which in turn vary as functions of the intensity and duration of the activity (Table 1). Basketball is about $20 \%$ aerobic and $80 \%$ anaerobic, and therefore many factors influence the exact energy expenditure ratio for individual players (Abdelkrim et al., 2007).

Assigning exact ratios to fit all styles of play would be impossible. It is widely accepted that basketball is a game requiring a high level of anaerobic fitness. This is certainly the case when a 2-hour game is broken down into shorter segments. For example, if we monitor one player for the first quarter $(10 \mathrm{~min})$, we can observe a work-rest ratio of 1:1 or less (Abdelkrim et al., 2007; Meckel and Gottlieb, 2009; Meckel et al., 2009), but if we monitor the same player for the whole game, we see a work-rest ratio of 1:2-1:3, given that the game includes short breaks: time-outs, quarter breaks and halftime (Gottlieb et al., 2014). While the energy to perform high-intensity efforts is derived primarily from the anaerobic system during the basketball game, recovery for subsequent bouts of exercise is facilitated during the rest periods by the aerobic system (Meckel and Gottlieb, 2009).

It is important to develop a training program that specifically emphasizes the energy 
system required to play basketball. Within $20 \mathrm{~s}$ of rest, $50 \%$ of the muscle stores of ATP-CP is restored, and $87 \%$ is restored after $60 \mathrm{~s}$. Heavy breathing after high intensity is the process through which the aerobic system metabolizes lactate in an effort to facilitate recovery. In addition, if basketball players have strong basic aerobic conditioning to tolerate high levels of accumulated blood lactate concentration, this will delay the onset of fatigue and enhance productivity on the court (Gottlieb et al., 2014; Meckel and Gottlieb, 2009; Meckel et al., 2009).

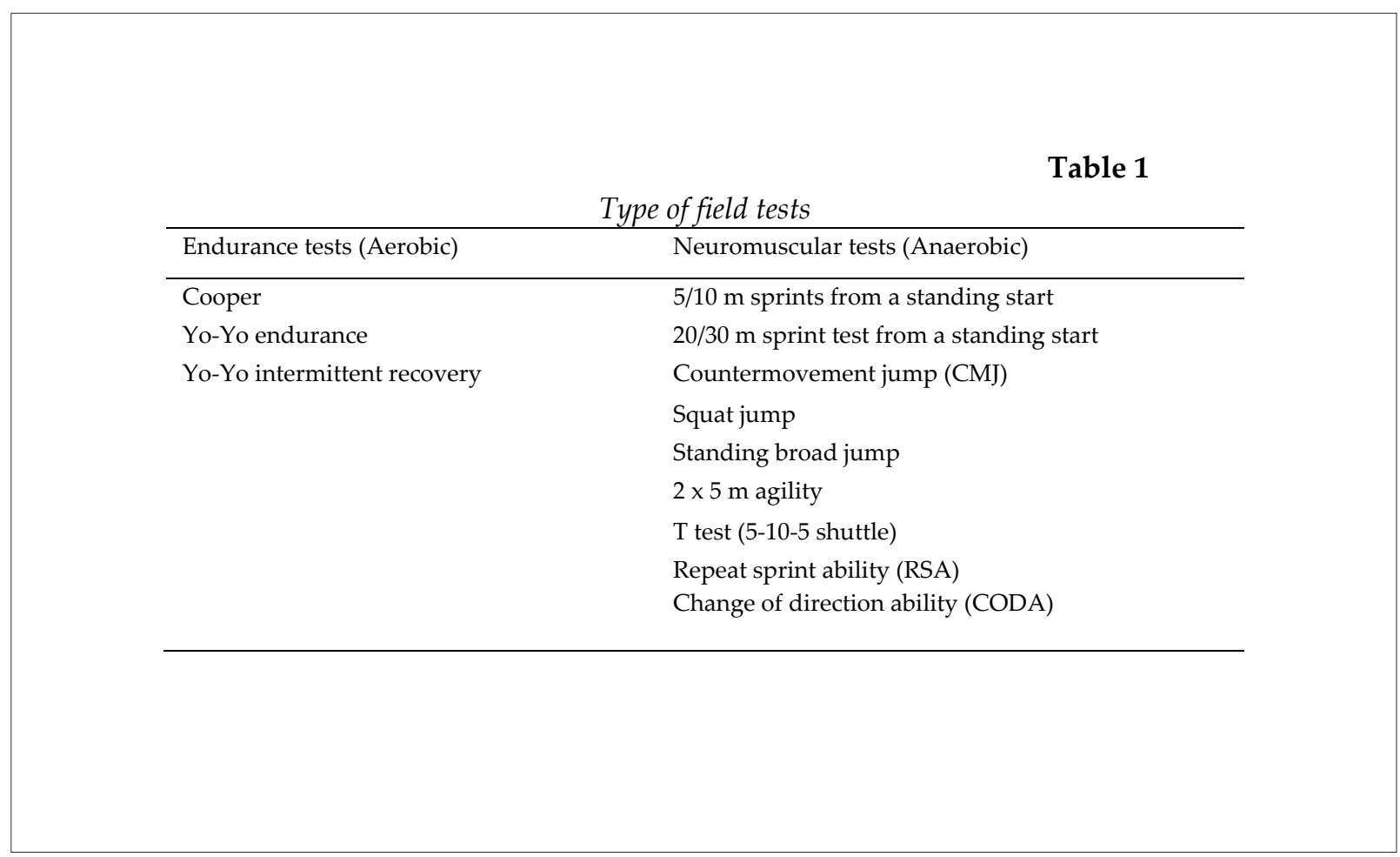

The physical requirements of a basketball game (intermittent exercise)

The last two decades have yielded a significant accumulation of specific data related to modern methods of coaching basketball (Shelling and Torres, 2016). The body structure and impressive athletic ability of basketball players may account for some of the rapid development of the sport in recent years (Delextrat and Cohen, 2008).

As noted, since the introduction of the 24s shot clock, the game has become much faster and the concept of fast playing has become crucial in basketball. This change has led to high physical demands on the players, both defensive and offensive, raising the importance of their explosive strength (Stojanovic et al., 2012). For this reason, strength and conditioning coaches and other professionals in the field seek more effective training methods for nurturing and developing players' physical abilities, as well as better methods to monitor and assess the fitness components required for the sport of basketball (Delextrat and Cohen, 2008; Meckel and Gottlieb, 2009).

Another important component required for repeated sprints is aerobic capacity for overall performance in the game. Nevertheless, most of the actions during the game are characterized as anaerobic, e.g. jumping, changing direction and footwork (Abdelkrim et al., 2007; Gottlieb et al., 2014). A basketball game is considered anaerobicdominated and requires repetitive short and intense sprints from the players. Such activities take a high toll on the players (Castanga et al., 2005, 2008). In a basketball game a player averages 105 intense movements lasting between 2 to $6 \mathrm{~s}$, which occur on average every $21 \mathrm{~s}$ on the game clock (not including time-outs). Intensity 
during these movements shows values of 60 to $75 \%$ of $\mathrm{VO}_{2 \max }$, and $70-90 \%$ of the maximum heart rate (Meckel and Gottlieb, 2009; Meckel et al., 2009). The overall distance a player sprints during the game is less than $10 \%$ of the total distance a player moves throughout a full game. Overall, the intermittent activity pattern in basketball demands aerobic capabilities sufficient to sustain repeated short bouts of high-intensity exercise (Bishop, 2004).

Despite the infrequency of these sprints, they have a great impact on the outcome of the game (Wragg et al., 2000). It should be noted that time-outs last for $2 \mathrm{~min}$, the halftime period for 15 $\mathrm{min}$, and foul calls from $20 \mathrm{~s}$ to $1 \mathrm{~min}$. The assessment of RSA as a training and research tool is also discussed (Spencer et al., 2005).

To repeat these activities without fatigue two main processes are required: 1) faster renewal of $\mathrm{CP}$ stores, and 2) faster removal of blood lactate concentration from the muscles (Gottlieb et al., 2014). Sport intensities and movement patterns during men's basketball were investigated by videoing the movements of eight elite players and monitoring their heart rate and blood lactate responses during competition (Gottlieb et al., 2014; Meckel and Gottlieb, 2009). The results are expressed in "live time", which means actual playing time and "total time" which includes live time as well as all stoppages in play. The mean \pm SD frequency of all activities was $997 \pm 183$, with a change in the movement category every $2.0 \mathrm{~s}$ (Ostojic et al., 2006). A mean total of $105 \pm 52$ highintensity runs (mean duration of $1.7 \mathrm{~s}$ ) was recorded for each basketball game, resulting in one high-intensity run every $21 \mathrm{~s}$ in live time. Sixty percent of live time was spent engaged in lowintensity activity, while $15 \%$ was spent in highintensity activity. The mean heart rate during live time was $169 \pm 9$ beats $/ \mathrm{min}^{-1}(89 \pm 2 \%$ peak HR attained during laboratory testing); $75 \%$ of live time was spent with an HR response greater than $85 \%$ of the peak heart rate. Mean blood lactate concentration was $6.8 \pm 2.8 \mathrm{mM} / \mathrm{L}$, indicating the involvement of glycolysis in the energy demands of basketball. It was concluded in these studies that physiological requirements of men's basketball are high, placing considerable demands on the cardiovascular and metabolic capacities of players (Abdelkrim et al., 2007; Ostojic et al., 2006).

Physical fitness is a performance factor that is characterized by its ability to be assessed using closed tests. Among the existing tests today, there are no data on which are the most optimal ones for the sport to which they are applied (Mancha-Triguero et al., 2019).

Tests for assessing aerobic and neuromuscular capacity

The aim of fitness tests is to assess the condition of athletes in terms of each fitness component, in order to determine what needs to be improved through the training program and to conduct retests at set times to assess whether their condition has changed. These tests are especially important among children and teenagers, so that coaches can see whether players are developing in terms of physical fitness as they get older (Chiu et al., 2003; Gottlieb et al., 2014; Hoffman, 1996; Mujika et al., 2009).

The ability to produce great power in a short period of time is an important measure in many sports, such as basketball, soccer and volleyball. For this reason, these team sports place great emphasis on improving strength at every age and every level of performance (Gottlieb et al., 2014). Optimal development and improvement of this ability, as well as of speed, agility and coordination, is not merely a theoretical exercise in comprehending the principles of physiology and training underlying these fitness components. It is also connected to the need for valid and reliable measurement techniques that make it possible to assess different abilities accurately and consistently (Delextrat and Cohen, 2008).

The most commonly used field tests in the basketball literature

The literature offers a number of widely used tests to measure aerobic and anaerobic fitness (Abdelkrim et al., 2007; Delextrat and Cohen, 2008; Gottlieb et al., 2014). The following are examples of field tests that can provide fitness coaches with relevant information for basketball:

\section{Endurance tests (aerobic):}

Yo-Yo endurance test - a maximum aerobic capacity test that includes running back and forth for $20 \mathrm{~m}$ with increasing effort until the participant becomes exhausted. The test has a high correlation with $\mathrm{VO}_{2 \max }(\mathrm{r}=0.92)$. This test is reliable and valid for predicting aerobic capacity in different populations (Clair et al., 1998). The test was chosen to evaluate aerobic fitness every few months, due to its suitability to the activity 
patterns routinely performed by athletes. The pace starts at $8 \mathrm{~km} / \mathrm{h}$ and increases by $0.5 \mathrm{~km} / \mathrm{h}$ every minute. The pace is dictated and made audible by an audio disc. Test results are determined by the number of times the athlete performs the sprints before reaching exhaustion (Castagna et al., 2005; Delextrat and Cohen, 2008; Ostojic et al., 2006).

Yo-Yo recovery test (Level 1) - this version of $Y_{0}-Y_{0}$ has a correlation of $\mathrm{r}=0.77$ with maximum $\mathrm{VO}_{2 \max }$. Assessment includes a $40-\mathrm{m}$ run, divided into $20 \mathrm{~m}$ up and $20 \mathrm{~m}$ back to the starting point, and $10 \mathrm{~s}$ recovery after each full cycle $(40 \mathrm{~m})$. The test starts at $10 \mathrm{~km} / \mathrm{h}$ and increases by $0.5 \mathrm{~km} / \mathrm{h}$ after each cycle. This version was developed for sports that require intense physical efforts followed by periods of incomplete recovery, such as basketball (Bangsbo, 2006; Castagna et al., 2005; Delextrat and Cohen, 2008).

Cooper test - in this test the player needs to run as far as he/she can in 12 minutes. Its predictive ability of $\mathrm{VO}_{2 \max }$ is very good $\mathrm{r}=0.89$ (Cooper, 1968), but does not reflect the character of all ball games (Castagna et al., 2005).

\section{Neuromuscular tests (anaerobic):}

5/10- $m$ sprints from a standing start - Starting Speed test: this test evaluates horizontal power while performing a cyclical movement - sprints from a standing start. The first step is decisive in the examinee's achievement. Times are usually measured by photo-electric cells or Optojump (Microgate, Italy). Each player performs 2 sprints from a high start, with 3-5 min of rest between runs. The best time is recorded (Balciunas, 2006; Gottlieb et al., 2014; Hoffman, 1996; Shaher, 2011).

20/30-m sprint test from a standing start Absolute Speed test: this test also evaluates horizontal power while performing a cyclical movement - sprints from a standing start. The ability to accelerate is decisive for good results. Times are usually measured by photo-electric cells or Optojump). Each player performs two sprints from a standing start, with 3-5 min of rest between runs. The best time is recorded (Delextrat and Cohen, 2008; Gottlieb et al., 2014; Hoffman, 1996; Mujika et al., 2009; Shaher, 2011).

Repeat Sprint Ability (RSA)Test - field-based team sports present some fitness components which are poorly understood. In particular, repeated-sprint ability (RSA) is one area that has received relatively little research attention until recent times. However, with improvements in technology, time-motion analysis has allowed researchers to document the detailed movement patterns of team-sport athletes. This type of practice involves the metabolic changes occurring during this type of exercise, such as energy system contribution, adenosine triphosphate depletion and resynthesis, phosphocreatine degradation and resynthesis, glycolysis and glycogenolysis, and purine nucleotide loss. Assessment of RSA, as a training and research tool, is also discussed (Spencer et al., 2015). The recognition of the important role of RSA for performance in basketball has led to the wide use of RSA-based tests among basketball players as part of routine fitness testing (Caprino et al., 2012; Meckel et al., 2009), as well as for training session strategy (Attene et al., 2015).

Countermovement jump (CMJ) test - this test assesses vertical power in a single jump. Jumps begin while standing straight, then knees are bent and quickly extended while leaving the ground and rising to the maximal height. Hands are usually placed on the hips in order to neutralize momentum from the arms. Each athlete performs 3 maximal jumps with about 2 min of rest between jumps. Jumps are usually performed using Optojump, which is connected to a digital timer that converts time in air to the jump height, or a force plate which contains sensors that measure the strength exerted by the feet (Gottlieb et al., 2014; Hoffman, 1996; Shaher, 2011).

Squat jump test - in the same way, it is possible to perform a squat jump as a test. Players assume a low squat position. Movement is stopped and then the athlete jumps as high as they can from this position, with hands on hips or behind their back (García-López et al., 2005).

Standing broad jump - athletes stand with both legs together. They bend their knees and use arm momentum in order to jump. The longest jump of 3 attempts is recorded as the result. If an athlete falls backward the jump must be repeated. Although the standing broad jump was part of the Olympic Game athletics competitions from 1900 to 1912, it has not been part of regular competitions worldwide for more than a century. In addition, no extensive statistics are available about achievements and progress in this event. If this test is used for assessing power, it is only by basketball clubs which do not have advanced equipment.

Also available are agility tests that assess 
changes of direction which require, among other things, great explosive strenght. Players need starting speed, a fast takeoff, stopping, acceleration, change of direction and at times sideways running. In addition, it is important to mention that the ability to change direction requires high technical skills combined with unique leg work (Sheppard and Young, 2006; Wragg et al., 2000). Many agility tests can be found in the literature. The two mentioned here are among the most accepted for assessing the explosive strength of basketball players (Delextrat and Cohen, 2008):

$2 \times 5-m$ agility test: this test measures sprinting time, turning, and changing direction. The test is performed by running $5 \mathrm{~m}$ in one direction, turning quickly, and returning $5 \mathrm{~m}$ to the starting point, a total of $10 \mathrm{~m}$ of running. The test can use photoelectric cells or an Optojump system to assess performance (Delextrat and Cohen, 2008; Sheppard and Young, 2006; Wragg et al., 2000).

T-test (5-10-5 Shuttle): this is an agility test that measures how quickly athletes can accurately change direction. The T-test is designed to measure lateral speed as well as forward and backward speed (Sheppard and Young, 2006; Wragg et al., 2000; Young et al., 2001).

\section{Conclusions}

The new generation of basketball players consists of highly conditioned athletes who ultimately elevate the level of the game (Spencer et al., 2005). For consistency in season-long, highlevel performance, conditioning is the key. Participation in youth sports such as basketball offers many potential benefits for children and adolescents (DeFiori et al., 2018). However, there is also a concern that an excessive focus on sportspecific intensive training and competition at a young age may impede an athlete's ability to develop transferable athletic skills, and possibly increase the risk of burnout and overuse injury (DeFiori et al., 2014).

Based on the literature review of field tests presented above, the impression is that sports researchers still seek specific tests to reflect as closely as possible abilities of athletes required in the game itself.

The activity of basketball players is based on a combination of horizontal movements (sprints and changes of direction), vertical movements (jump shots and rebounds), and movements that combine the two movement planes, mainly when penetrating to the basket and blocking shots. These are high-intensity movements that are usually performed intermittently throughout the game and at different intervals, by players in the various positions on the court (Gottlieb et al., 2014).

This combined type of movement has caused fitness coaches, physiologists, and other professionals in the field to seek the most effective training methods for promoting and developing physical abilities in basketball players (Delextrat and Cohen, 2008; Gottlieb et al., 2014). Today, as fitness coaches deliberate long and hard about which training method is most effective for developing explosive strenght, they encounter many limitations in choosing the most effective tests for assessing this component. A number of efforts have been made to develop specific tests for assessing power among players (Delextrat and Cohen, 2008; Ostojic et al., 2006; Sheppard and Young, 2006; Wragg et al., 2000). Studies show a correlation between horizontal and vertical power (Hori et al., 2008; Sheppard et al., 2008). However, the literature does not offer a sufficient number of specific tests in ball sports to enable examining power where horizontal and vertical movements are combined (Gottlieb et al., 2014).

Karcher and Buchheit (2016) conducted a study in team handball which examined the benefit of conducting $\mathrm{CMJ}$ tests to predict specific jumping ability in handball. The results showed no clear correlation between time in the air in jump shots and the $\mathrm{CMJ}$, indicating that the use of the $\mathrm{CMJ}$ for predicting specific jumping ability in handball, with its combination of horizontal and vertical movement, is questionable. Thus, specific tests are needed that reflect jumping ability and power for team handball. In contrast, a volleyball study by Sheppard et al. (2008) revealed a strong connection between CMJ performance and the jump serve which combines both horizontal and vertical components as does the spike jump. This seems to indicate that specific connections among these variables may differ from one sport to another and between the various developmental levels of athletes. However, it is still not known whether the protocols of tests like the CMJ can actually predict specific jumping abilities in basketball (such as the actual jumping time when penetrating to the basket while holding the ball and pushing off from one leg). As for improvement in performance, 
several intervention studies in ball games have shown that maximal strength training, plyometric training, sprint training, complex training, and other types of training all significantly improved CMJ performance (DeFiori et al., 2014; Gottlieb et al., 2014; Mujika et al., 2009). At the same time, it is still not clear whether these improvements can be transferred to game situations, such as lay-ups and penetrating to the basket, and therefore there is a need for future studies.

There is no doubt that aerobic capacity is significant in basketball for rapid recovery from sprints and repeated jumps (Meckel et al., 2009). The competition demands encountered by basketball players suggest that both anaerobic and aerobic energy pathways contribute to energy sources (Edwards et al., 2018), which cause fatigue and lower the rate of activity, thus impinging on the quality of a player's game. Therefore, coaches and fitness coaches must correctly measure their players' aerobic capacity, mainly before the season begins. Today a number of tests for aerobic capacity are available, both laboratory and field tests (Gottlieb et al., 2014; Meckel and Gottlieb, 2009). The laboratory $\mathrm{VO}_{2 \max }$ test is accurate, but because of its high cost various field tests, such as the Cooper test (sequential 12-min running tests), have been developed (Cooper, 1968).

Various types of Level 1 Yo-Yo tests (Bangsbo, 2006; Delextrat and Cohen, 2008) yield good predictions of $\mathrm{VO}_{2 \max }$ (aerobic ability) in ball games defined as sports with intermittent activities - in other words, many intense activities with short recovery periods. However, the tests are not adapted specifically to particular sports. In other words, the specific elements of the sport, as well as rest periods, changes of direction, typical movements, etc., must be taken into consideration when adapting the tests for particular sports. In general, the transfer of physical improvements from training to team ball games is difficult to predict, when technical abilities, complex interactions between players, and other factors must be considered.

\section{Future lines of research}

One of the problems with many of the physiological and biomechanical studies of the vertical jump is that they neutralize arm movement in an attempt to isolate the effect of leg muscle power in the action. In this way they hope to find a causal relationship between improved muscular power or muscle output, and jump height. Yet it must be kept in mind that few sports require athletes to jump without arm momentum (i.e., with arms on hips or behind the back). The result is that such tests are less useful for coaches in the field. In general, it is advisable to adhere to the principle of specificity in training - improving a given motor skill should entail practicing that skill as it is performed in games/competitions. To this end, specific tests for basketball should be developed and used.

One of the aspects to be considered is recovery time. Sports in general, and basketball in particular, involve intense but non-consistent body movement. Based on the observation and analysis of 15 basketball games in the Israel Premier League, it is apparent that basketball players experience incomplete recovery during rest periods that last from $10 \mathrm{~s}$ (for fouls with no free throws) and $30 \mathrm{~s}$ (for fouls with two free throws) to time-outs of about one minute in duration, and timeouts of somewhat more than two minutes between quarters. Importantly, the frequency of recovery (10-30 s) was observed on average every three possessions.

To the best of our knowledge, no specific test has yet been developed to assess the unique features of basketball. Thus, it would be both important and interesting to build a reliable and valid test of which results will provide coaches with tools for testing the specific abilities required in basketball, even if certain compromises must be made to accommodate field-based limitations.

The reviewed aerobic tests demonstrate a good correlation with $\mathrm{VO}_{2 \max }$ (Meckel and Gottlieb, 2009; Meckel et al., 2009) which is very important for recovery from short, intense bouts of movements of a few seconds. However, they do not offer elements similar enough to basketball movements which require leg muscle exertion. A basketball-oriented test should take this into consideration as well. The analyzed bibliography reveals the lack of the design and use of specific tests to highlight the qualities involved in the targeted sport (Mancha-Triguero et al., 2019). 


\section{References}

Abdelkrim N, Fazaa S, Ati J. Time motion analysis and physiological data of elite under 19 year old basketball players during competition. J Strength Cond Res, 2007; 24: 2330-2342

Alemdaroğlu U. The relationship between muscle strength, anaerobic performance, agility, sprint ability and vertical jump performance in professional basketball players. J Hum Kinet, 2012; 31: 149-158

Attene G, Pizzolato F, Calcagno G, Ibba G, Pinna M, Salernitano G, et al. Sprint vs. intermittent training in young female basketball players. J Sports Med Phys Fitness, 2014; 54: 154-161

Balciunas M, Stonkus S, Abrantes C, Sampaio J. Long term of different training modalities on power, speed, skill and anaerobic capacity in young male basketball players. J Sport Sci Med, 2006; 5: 163-170

Bangsbo J. Fitness Testing in Football: 1-200; 2006

Ben Abdelkrim N, El Fazaa S, and El Ati J. Time-motion analysis and physiological data of elite under-19-yearold basketball players during competition. Br J Sports Med, 2007: 41: 69-75. 10.1136/bjsm.2006.032318

Bishop D. The effects of travel on team performance in the Australian national netball competition. J Sci Med Sport, 2004; 7:118-122.

Caprino D, Clarke ND, and Delextrat A. The effect of an official match on repeated sprint ability in junior basketball players. J. Sports Sci, 2012; 30: 165-1173

Castagna C, Grant A, Stefano D. Competitive-level differences in yo-yo intermittent recovery and 12-minute run test performance in soccer referees. J Strength Con Res, 2005; 19: 805-809

Castanga C, Grant A, Vincenzo M, Giueseppe A, Evira P, Stefano D. Effect of recovery mode on repeated sprint ability in young basketball players. J Strength Cond Res, 2008; 22: 1-7

Chiu L, Fry L, Weiss B, Schilling L, Brown S. Post activation potentiation response in athletic and recreationally trained individuals. J Strength Cond Res, 2003; 17: 671-677

Clair G, Broomhead S, Lambert M, Prediction of maximal oxygen uptake from $20 \mathrm{~m}$ shuttle run as measured directly in runners and squash players. J Sport Sci, 1998; 16: 331-335

Cooper M. A means of assessing maximal oxygen intake correlation between field test and treadmill testing. JAMA, 1968; 203: 201-204

Crisafulli A, Melis F, Tocco, P. Laconi, P Lai, and Concu . External mechanical work versus oxidative energy consumption ratio during a basketball field test. J Sports Med Phy Fit, 2002; 42:409-417

DeFiori JP, Benjamin HJ, Brenner J, Gregory A, Jayanthi N, Landry GL, Luke A. Overuse injuries and burnout in youth sports: a position statement from the American Medical Society for Sports Medicine. Clin J Sport Med, 2014; 24:3-20

DeFiori JP, Gülich A, Brenner JS, Côté J, Hainline B, Ryan E 3'r, Malina RM. The NBA and youth basketball: Recommendations for promoting a healthy and positive experience. Sports Med, 2018; 48: 2053-2065

Delextrat A, Cohen D. Physiological testing of basketball players: towards standard evaluation of anaerobic fitness. J Strength Cond Res 2008; 22: 1066-1072

Edwards T, Spiten T, Piggott B, Bonhotal J, Haff G, Gregory JC. Monitoring and managing fatigue in basketball. Sports (Basel), 2018; 6: 19

Garcia-Lopez J, Peleteiro J, Rodriguez-Marroyo JA, Morante JC, Herrero JA, Villa JG. The validation of a new method that measures contact and flight times during vertical jump. Int J Sports Med, 2005; 26: 294-302

Glaister M. Multiple sprint work. Sport Med, 2005; 35: 757-777

Gottlieb R, Eliakim A, Shalom A, Iacono AD, Meckel Y. Improving anaerobic fitness in young basketball players: Plyometric vs. specific sprint training. J Ath Enhance, 2014; 3: 3. doi:10.4172/2324-9080.1000148

Hoffman JR. Relationship between athletic performance tests and playing time in elite college basketball players. J Strength Cond Res, 1996; 10: 67-71

Hori N, Newton, RU, Andrews WA, Kawamori N, McGuigan, MR, Nosaka, K. Does performance of hang power clean differentiate performance of jumping, sprinting and changing of direction. J Strength Cond Res, 2008; 22: 412-418

Karcher C, Buchheit M. Shooting performance and fly time in highly trained wing handball players: Not everything is as it seems. J Sports Physio Perform, 2016; 24: 1-20 
Mancha-Triguero D, García-Rubio J, Calleja-González J, Ibáñez SJ. Physical fitness in basketball players: a systematic review. J Sports Med Phys Fitness, 2019 Sep; 59(9): 1513-1525.

McInnes SE, Carlson JS, Jones CJ, McKenna MJ. The physiological load imposed on basketball players during competition, J Sports Sci, 2008; 13: 387-397

Meckel Y, Gottlieb R. Repeated sprint tests in young basketball players at different game stages. Eur J App Physiol, 2009; 107: 273-279

Meckel Y, Machnai O, Eliakim A. Relationship among repeated sprint test, aerobic fitness and anaerobic fitness in elite adolescent soccer players. J Strength Cond Res, 2009; 23: 163-169

Mujika I, Santisteban J, Castagna C. In-season effect of short-term sprint and power training programs on elite junior soccer players. J Strength Cond Res, 2009; 23: 2581-2587

Narazaki K, Berg KE, Stergiou N, Chen B. Physiological demands of competitive basketball. Scand. J Med Sci Sport, 2009; 19: 425-432

Ostojic SM, Mazic S, Dikic N. Profiling in basketball: Physical and physiological characteristics of elite players. J Strength Cond Res, 2006; 20: 740-744

Shalfawi SA, Sabbah A, Kailani G, Tønnessen E, Enoksen E. The relationship between running speed and measures of vertical jump in professional basketball players: A field test approach.J Strength Cond Res, 2011; 25: 3088-3092

Shelling X, Torres L. Accelerometer load profiles for basketball-specific drill in elite players. J Sports Sci Med, 2016; 15: 585-591

Sheppard JM, Cronin JB, Gabbett TJ, McGuigan MR, Etxebarria N, Newton RU. Relative importance of strength, power and anthropometric measures to jump performance of elite volleyball players. J Strength Cond Res, 2008; 22: 758-765

Sheppard JM, Young WB. Agility literature review: classification, training and testing. J Sports Sci Med, 2006; 24: 919-932

Spencer M, Bishop D, Dawson B, Goodman C. Physiological and metabolic responses of repeated-sprint activities: specific to field-based team sports. Sport Med, 2005; 35: 1025-1044

Stojanovic MD, Ostojic SM, Calleja- González J, Milosevic Z, Mikic M. Correlation between explosive strength, aerobic power and repeated spring ability in elite basketball players. J Sports Med Phys Fit, 2012; 52: 375381

Stolen T, Chamari K, Castagna C, Wisloff U. Physiology of soccer: An update. J Sport Med, 2005; 35: 501-536

Wragg CB, Maxwell NS, Doust JH. Evaluation of the reliability and validity of a soccer specific field test of repeated sprint ability. Eur J App Physiol, 2000; 83: 77-83

Young WB, McDowell MH, Scralett NJ. Specificity of sprint and agility training methods. J Strength Cond Res, 2001; 15: 315-319

\section{Corresponding author:}

\section{Roni Gottlieb M.P.E,}

The Academic College at Wingate,

Wingate Institute, Netanya, Israel;

Phone: +972 (0) 9 7417844;

Cellular: +972 544822425

E-mail: ronigot23@gmail.com 